\title{
Improving induction of labour - a quality improvement project addressing Caesarean section rates and length of process in women undergoing induction of labour
}

Sabrina O'Dwyer, Caterina Raniolo, Janice Roper, Manish Gupta

Barts Health NHS Trust, United Kingdom

\begin{abstract}
Induction of labour (IOL) in maternity care is often not an area of priority in maternity services, which often results in protracted delays, a poor patient experience, and patient complaints. Caesarean section (CS) rates among women undergoing IOL at this inner city district general hospital were noted to be higher than other units nationwide.
\end{abstract}

We collected pre and post-intervention data of the following outcome measures: time taken to administer prostaglandin after arrival, time taken to achieve established labour, mode of delivery, and user satisfaction scores. Our introduction of a dedicated IOL Suite, promotion of outpatient IOL, use of a single administration prostaglandin (as opposed to traditional six hourly prostaglandin), widespread staff engagement and rolling audit has resulted in positive change in the maternity unit. CS rates for women undergoing IOL have been reduced from $29 \%$ to $22 \%$ $(p=0.05)$, time taken to administer the induction medication has decreased from $6.3 \mathrm{~h}$ to $2.7 \mathrm{~h}(\mathrm{p}=0.0001)$, and out-patient induction rates have increased from $3 \%$ to $33 \%(p=0.001)$.

We have achieved a reduction in the overall length of in-patient stay. We have also received positive feedback from both staff and patients. We used a bottom-up approach, engaging frontline staff in problem identification and pathway design. Our staff engagement questionnaire showed other benefits such as increased staff morale as a result.

Collection of simple performance data and sharing of this in real time with staff acts as a valuable tool for acceptance of change and continuous improvement. Communicating plans to a large body of people is important in ensuring the success of an intervention. Staff showing disengagement may require specific detailed information to allay their concerns. Following initial successes, ongoing vigilance, and collection of audit data is key to sustaining any improvement.

\section{Problem}

Our maternity unit is set in an inner city UK district general hospital with mixed socio-economic demographic and complex patient needs due to a multi-ethnic population with a high proportion of women born outside the UK. The annual delivery rate is about 5000 deliveries per year.

We identified IOL as a potential area for improvement work in the maternity unit. Patient complaints frequently described a poor experience during IOL. There was a need to measure baseline performance and to formally assess potential for improvement.

There had already been some attempts in the year prior to commencement of this project, to introduce outpatient IOL for lowrisk women being induced for post-maturity. However, this was not successful and uptake was low. There had also been some attempts to introduce slow release dinoprostone pessaries as an induction agent, but these had been abandoned in favour of prostaglandin gel. Reasons for the initial unsuccessful introduction of slow release dinoprostone were not formally investigated or documented, but anecdotally were concerned with staff's lack of familiarity with the drug, incorrect administration, and unwillingness to adopt the change.

Criticisms of previous improvement efforts included the use of a topdown approach to implementation, without involvement of frontline staff in pathway design. The recent merger of maternity units to form a larger trust had also caused friction between management and frontline staff.

\section{Background}

With a rise in incidence of obesity and advanced maternal age, as well as increasingly complex medical needs of pregnant women in the UK, induction of labour (IOL) rates have shown a gradual increase of about $1 \%$ each year. Latest figures from 2013 put induction rates at $23.3 \%$.[1]

IOL represents a significant workload on maternity units and there is wide variation in practice. A report published by the Royal College of Obstetricians and Gynaecologists on patterns of maternity care in English units in 2011 to 2012 showed that emergency Caesarean section (CS) rates following IOL ranged from $15.6 \%-45.6 \%$ among primiparous women.[2] This variation can be explained at least in part by inconsistencies in clinical 
management of $\mathrm{IOL}$ that exists from unit to unit.

Randomised controlled trial data has shown that out-patient IOL results in higher patient satisfaction scores, $[3,4]$ and theoretical benefits include a shorter inpatient stay, decreased staff workload, and resulting economic savings.[5,6]

With regards to the type of Prostaglandin medication used in IOL, two commonly used medications include a gel that is administered six-hourly into the vagina, and a 24 hourly slow release pessary, also administered via the vaginal route. The slow release dinoprostone vaginal pessary releases $0.3 \mathrm{mg}$ of Prostaglandin per hour after a single administration, up to a maximum dose of $10 \mathrm{mg}$ per pessary. A single pessary is meant to be left in situ for 24 hours. There are no differences in efficacy of pessary versus gel in terms of induction-to-delivery times, CS rates, and neonatal outcomes.[7] Benefits of the pessary include fewer vaginal examinations and relatively less midwifery workload as it eradicates the need for multiple episodes of electronic fetal monitoring and repeated vaginal examinations.

\section{Baseline measurement}

As a first step we gathered information, both qualitative and quantitative about the current state of the service. Quantitative measurements were done with retrospective and prospectively collected audit data. 60 IOLs were studied:

1. Time taken between admission and administration of the first prostaglandin gel (mean $=6.3 \mathrm{~h}$ )

2. Time taken to achieve established labour (mean $=31.3 \mathrm{~h}$ )

3. Type of IOL (3\% out-patient, $97 \%$ in-patient)

4. Place of labour ( $0 \%$ birth centre, $100 \%$ labour ward).

Histograms of logarithmic values were plotted to ensure normality in data for time taken.

Baseline Caesarean section rates were determined by looking at the birth register of all births in November 2013 to January 2014 (three month period). Through the birth register we identified all the IOLs, and could determine the mode of delivery for each. The CS rate was $29 \%$ in 212 of the IOLs carried out.

Qualitative data collected included:

1. Patient collected data detailing a process map of the patient journey through an induction of labour

2. Patient satisfaction survey prospectively distributed to all women undergoing induction of labour during audit period, and a retrospective survey distributed to women on local National Childbirth Trust and Maternity Service Liaison Committee electronic mailing lists

3. Collecting staff perceptions of the problems in the current process of induction of labour via face to face informal interviews, suggestion boards left in staff common rooms, and via a workshop

4. Conducting a staff engagement questionnaire.
Our process map of a patient's experience described how a woman received one dose of prostin gel in a three day period. There was a protracted delay in transferring her to labour ward for the next step of the IOL (amniotomy). When she finally had the amniotomy, she delivered her baby a few hours afterwards.

Patient satisfaction surveys told us that women were unhappy about the length of time taken for IOL, and that they felt uninformed and often unsupported during the process, particularly when there were delays. Staff felt that long waiting times for commencement of $\mathrm{IOL}$, and frequent delays in continuing $\mathrm{IOL}$ were common problems potentially leading to a high CS rate. These inefficiencies caused a lot of frustration among service users as well as caregivers.

\section{Design}

We supported staff to deliver change by sending senior staff to a two-day NHS Staff College course, aimed at exploring teamworking, trust dynamics, and morale issues within the unit.

Following this, a workshop involving frontline staff performed a gap and root-cause analysis using all the information collected above. The root causes for the delays and problems surrounding the process of induction of labour identified were:

- Inconsistent, out-dated and impractical guidelines

- Poor patient information

- Lack of unit wide coordination

- Ineffective use of staff and resources for induction

- No feedback to staff - good or bad

- Lack of clarity about roles and responsibilities.

A second workshop to design the desired future state, also involved frontline staff of all levels. Changes were required to improve the patient experience primarily through reducing delays and overall time needed to achieve active labour. Ideas for improvement suggested during patient and staff questionnaires were incorporated into our intervention.

The newly designed induction of labour process included the following features:

- The replacement of prostaglandin gel that had to be administered six-hourly, with single administration slow release dinoprostone pessaries

- The setting up of a dedicated IOL suite where IOLs are to be carried out, with a dedicated midwife and IOL champions promoting good practice

- Promoting outpatient IOL as normal practice for low risk women

- Promoting the birthing unit as the appropriate birthing venue for 
low risk women who go into labour after dinoprostone alone

- Allowing partners to stay with women having IOL as inpatients overnight

- Aiming for women to be on the labour ward 24 hours after insertion of dinoprostone pessary so that timely amniotomy can be performed, followed by oxytocin infusion if needed

- Introduction of an information centre on the induction suite where realtime performance indicators can be recorded to give staff feedback

- Streamlining and simplifying the induction of labour process by agreeing on a uniform guideline, minimise variation in practice, and to provide staff with clear guidance as to where women should be induced, how they should be induced, when they should be transferred to labour ward, when artificial rupture of membranes should be carried out, and how women with a previous caesarean section should be induced, including the use of double balloon catheters

- Writing a new induction of labour information leaflet and distributing to patients in the antenatal clinic. The leaflet was to be written in collaboration between midwives, doctors and service users.

A structured implementation plan was then derived and delivered, again with the involvement of key front line staff. All changes were carried out without need for additional staff or budget requirement, as our intention was for the changes to be sustainable in the long term. A launch date of 21/7/2014 was agreed upon.

\section{Strategy}

PDSA cycle 1: We took a sample of women over two weeks and identified 10 women who fulfilled the criteria for out-patient IOL. One hundred percent of women who fulfilled the criteria were discharged for a part of the IOL. We also found that another woman in that timescale also had outpatient IOL. She was being induced at 40 weeks for advanced maternal age and had no other risk factors. She requested outpatient IOL and after counselling by a doctor, had an outpatient IOL. We amended that guideline to say that outpatient IOL could be offered to women with advanced maternal age, as well as women being induced for gestational diabetes that was well controlled with diet alone (after appropriate counselling).

PDSA cycle 2: We wished to test if women were receiving the dinoprostone pessary within two hours of arriving on the IOL suite. Out of 10 women studied, eight women received the pessary within our target two hours. Reasons for the delay in administration were:

- Lack of dedicated IOL midwife due to staffing shortages

- Needing to wait for a doctor to prescribe the medication

- Labour Ward telling staff on IOL suite not to start IOLs as labour ward was too busy
- Some women were assessed to be favourable for amniotomy and hence the pessary would not be given. However, as beds were often short on labour ward for amniotomy, the woman would wait many hours for her IOL to commence (by amniotomy or otherwise).

In response to these observations, we recognised that ongoing negotiations with regards to staffing levels were being carried out. We also prepared IOL packs for the antenatal clinic, with blank drug charts and leaflets, in order to remind doctors to prescribe the medication in advance, and to distribute the leaflets. We used our various communication tools, including a "message of the week" to change the culture of IOLs being delayed due to high workload on labour ward, and specifically mentioned in the new guideline that this should not happen. Finally, we also amended the guideline to say that as long as a woman was not already contracting, she should have the pessary even if she is favourable for amniotomy, so as not to delay the commencement of the IOL.

PDSA cycle 3: We wished to see if women were being transferred to labour ward/birthing unit within 30 minutes of fulfilling the criteria for transfer (either they are in active labour, or the pessary has been in situ for 24 hours). Among 10 women who required transfer because they went into labour, eight out of 10 were transferred within 30 minutes. Among 10 women who required transfer for amniotomy (pessary being in situ for $24 \mathrm{~h}$ ), two of 10 were transferred within 30 minutes. Delays for transfer were often protracted (31 to 77 hours) in these cases. This highlighted the problem of the flow of women between the induction suite, labour ward, and the postnatal ward. Ongoing work is being done to tackle the team working and staffing problems the unit is facing. We also changed our protocol after reviewing experience from other units, in order to allow the pessary to remain in situ for a maximum of 32 hours total, as this may theoretically increase the change of women going into labour as the drug is still being released whilst they are waiting for a bed for amniotomy on labour ward.

\section{Results}

Use of electronic patient records in maternity care was introduced in May 2014, two months prior to introduction of our interventions in July 2014. Post intervention audit data was extracted from the electronic patient record, which minimised missing data because as long as we had the hospital number or name of any patient having induction of labour, we were able to obtain data sets. We identified the women undergoing IOL via the IOL booking system and corelated with the admission list for the IOL Suite.

We maintained a high standard of internal validity as all cases of IOL were identified via the list of cases prospectively recorded daily on the IOL suite information centre, and also via the IOL booking sheets. A dedicated improvement midwife, the second author (CR), was primarily responsible for the data collection process. First, second, and third authors had specific training in data collection and measurements for improvement, following the Institute for Healthcare Improvement model.[8]

Data were analysed using sequential run charts, with statistical process control (SPC) on key parameters: 
- Time taken for drug to be administered

- Time taken to achieve end-point (defined as active labour regular, strong contractions accompanied by cervical dilatation of $\geq 4 \mathrm{~cm}$ - or delivery if CS occurs prior to the onset of active labour).

Post-intervention data were collected from $100 \%$ of women identified as having undergone IOL between $21 / 7 / 14$ to $31 / 12 / 14$, with complete data collection made possible by access to electronic labour records, contemporaneously recorded into the electronic patient record system that was introduced in May 2014. We analysed 296 IOLs in this time period.

From $1 / 1 / 15$ to $31 / 6 / 15$, we collected data from a random sampling of IOLs each month. We analysed a further $65 \mathrm{IOLs}$ in this time period. Data were collected in monthly intervals after project commencement, so that monthly updates and feedback could be given to frontline staff.

Figure 1 shows a sustained increase in the uptake of outpatient IOL in the study period, from $3 \%$ pre-intervention to $33 \%$ postintervention ( $p=0.001)$. Post-intervention, we also had 10 women labour in the birthing unit, a change which although was not statistically significant, represented for us a paradigm shift as midwives were now considering women for delivery in the birthing unit if they were otherwise low risk and went into labour with the pessary alone (which is the recommended practice by the The National Institute for Health and Care Excellence).

Figure 2 shows how the intervention affected CS rates. The line graph shows the variation in monthly CS rates and the table (figure 3) shows numerical data for mode of delivery. Figure 3 also shows that the overall decrease in CS rate was attributable to the decrease in CS rate for primiparous women undergoing IOL. There was no difference in CS rate pre and post intervention for multiparous women.

Time taken to administer the induction medication has decreased from mean values of 6.3 hours to 2.7 hours ( $p=0.0001$ ). In considering the overall time taken to achieve our IOL end-point, defined here as a woman being in active labour (contracting regularly, cervix more than four centimetres dilated, and in the appropriate place for delivery), our mean baseline value was 31.3 hours. Post-intervention, our mean time taken from admission to end-point showed no significant change, at a mean of 28.0 hours $(p=0.4)$.

As supported by our PDSA cycles, we faced significant challenges in reducing the amount of time women waited for transfer to labour ward should they require amniotomy as part of the next stage of their IOL. It took time for us to reduce the overall IOL time over the intervention period. We achieved a mean 22.0 hours admission to end-point in the month of December through reinforcement of key messages and monthly positive and negative feedback to staff, , which was the last month for which we collected comprehensive data for all the IOLs carried out. In comparing data from December with the baseline, we did reach a statistically significant improvement $(p=0.05)$.
Figures 4 shows the run chart of the time taken from admission to administration of the prostaglandin, for IOLs arranged in sequential order. Statistical process control was applied, which shows a reduction in the mean and upper control limit for time from admission to prostaglandin administration. In figure 5 we have the run chart of the time taken from admission to end-point of the IOL, which does shows a small decrease in the mean post-intervention, but insufficient to demonstrate a significant change.

P-values for categorical data were calculated using chi-squared tests and two-tailed unpaired student's t-tests were used for numerical data. Time in minutes was transformed onto the log scale to ensure normality.

As a result of all these changes, small reductions in bed occupancy were achieved. Two main factors contributed to this:

1. Women receiving outpatient induction spent a mean of 14 hours at home which previously would have been spent in hospital

2. Fewer women had Caesarean Sections and therefore more were able to go home quickly following delivery.

The cumulative saving in bed occupancy on the combined anteand post-natal ward from these factors was calculated as 1.5 patients per day.

As seen in our baseline versus December 2014, bed occupancy would be reduced even further if we manage to sustain the effect of reduction in total time taken to achieve the end-point data.

See supplementary file: ds6044.docx - "Figures results"

\section{Lessons and limitations}

Our audit data were based on medical documentation, and it is possible that the timings reflected on medical documentation may represent some inaccuracies in the reflection of actual events. However, there was no other practical way in which to collect these data. Our data collection post intervention was more extensive than in most audits as we were able to extract all information from electronic patient records. This was due to the implementation of a fully paperless system of patient records in the maternity unit just prior to our project launch date.

Our baseline data showed a CS rate on the higher side of what is observed nationally, which might make our intervention less generalisable to units that are already performing well on the measure of CS rates amongst IOLs.

We also did not show any difference in CS rates when looking at multiparous women, and the overall decrease in CS rate was attributable to a decrease in CS rate for primiparous women in particular. In spite of this, we thought it would be beneficial to standardise the IOL process for both groups of women for simplicity, and to ensure compliance.

Our data for the multiparous group may also be influenced by the 
inclusion of women who had a previous CS. Unfortunately, we did not collect this information during the audit, and decided that retrospective data collection was not worthwhile due to small numbers of women having IOL with previous CS. Due to the added clinical complexities involved in IOL for women with a previous uterine scar (an increased risk of scar rupture with induction agents), we chose not to focus on this group during this project.

Widespread staff engagement and a bottom-up approach to designing the new pathways were crucial in ensuring the success of this project. We also employed a five-month consultation period with extensive root cause analysis, where we established staff morale and team working issues to underlie some of the problems with delays. These problems became more evident as the project went on, because even though we successfully reduced the time it took for the IOL to start after admission, we struggled for months after implementation with reduction of time in the transfer to labour ward for amniotomy after cervical ripening. The problem of patient flow between locations was due to the labour ward and antenatal/postnatal wards each protecting the time and workload of their own staff. Staff shortages are a general feature of day to day working, creating a fear of taking on too much work even if there was not any true staff shortage, and a bottleneck of patients in the pathway.

At the start of the project, we predicted that the high Caesarean section rate might be due to the long time it took from start of the induction to the achievement of active labour. Our data showed that there was a reduction in the Caesarean section rate preceding any notable decrease in overall induction of labour time. This may be due to the higher uptake of outpatient induction, where up to 24 hours of induction time is spent at home and hence less emotionally and physically draining for the woman.

The other reason may be due to a larger number of women going into labour in the first 24 hours due to sustained release of prostaglandin, as opposed to the staff dependent administration of prostaglandin gel used in our baseline data. Our study was never designed to be powered enough to measure statistically significant changes in accordance to research methodologies as it is a Quality Improvement study. Research conditions would have to be imposed in order to identify if any single intervention resulted in a lower CS rate. However, existing research in use of slow-release dinoprostone and out-patient IOL does not demonstrate this.[9,10]

We attribute the success of this improvement work to the following factors, which can be generalizable to any area of improvement:

1. The investment in a dedicated improvement midwife with allocated time to spend working on the project. She was able to concentrate on the communications strategy (message of the week, bulletin updates, face to face training, and mass emails), collect audit data, and publish updated performance graphs to staff on a monthly basis, give individualised feedback to staff members performing well or needing improvement, and to be on the shop floor working alongside ward staff when the IOL project was initially launched
2. The involvement of the trust service transformation team, which provided training in quality improvement methodology, and guidance in the application. As a result, data collection was robust, reliable, and convincing, in order to ensure continued support for the project by senior and junior staff, as well as management

3. Widespread engagement of frontline staff, including involvement of a junior doctor on the project core team, as well as early and continued engagement of key stakeholders. These stakeholders included senior management staff such as the medical director and clinical group director, as well as local patient advocacy groups and patients themselves.

Our main challenge for the future would be in sustaining the effects of our changes, particularly when we withdraw support from the improvement midwife dedicated to the project and cease our extensive audit efforts. We recommend ongoing spot checks and ongoing feedback to frontline staff on their individual and group performance. Our year long audit has taught us that sustaining the effects of change is an ongoing challenge, and that culture change takes a long time to embed.

\section{Conclusion}

Key features in our improvement work included the promotion of outpatient IOL, switch from six hourly prostaglandin gel to a singleadministration pessary that works over 24 hours, emphasis on timely administration of the prostaglandin pessary, and a move towards amniotomy $+/$ - oxytocin infusion as soon as possible following 24 hours of pessary use. We found these interventions effective in reducing CS rates and reducing the time taken to administer the prostaglandin after admission. There was a small reduction in time from admission to end point, which we hope will be sustained and further improved with more time.

$\mathrm{IOL}$ is an area of maternity care that involves staff members from across most sectors of maternity; from the booking and information process in antenatal clinic and in the community clinics, to the actual IOL process in the antenatal ward, to continuation of IOL, labour, and delivery on the delivery suite. Thus any implementation of change would affect large members of staff and challenge established working practices that were ingrained. A clear communications strategy is essential in conveying information in multiple different modalities, across a large staff population. A two way feedback system once the project is implemented is vital, for early troubleshooting. We also found that monthly publication of audit data was motivational for staff to continue to perform well, and vital to sustaining any improvement in the long term.

\section{References}

1. Health and Social Care Information Centre. The NHS Maternity Statistics, England: 2012-13. 5/12/13; Available at: http://www.hscic.gov.uk/catalogue/PUB12744. Accessed 5/29, 2015.

2. Knight H, Cromwell D, Van der Meulen J, Gurol-Urganci I, 
Richmond D, Mahmood T, et al. Patterns of maternity care in English NHS hospitals 2011/12. RCOG Press 2013.

3. Biem SR, Turnell RW, Olatunbosun O, Tauh M, Biem HJ. A randomized controlled trial of outpatient versus inpatient labour induction with vaginal controlled-release prostaglandin-E2: effectiveness and satisfaction. J Obstet Gynaecol Can 2003 Jan;25(1):23-31.

4. O'Brien E, Rauf Z, Alfirevic Z, Lavender T. Women's experiences of outpatient induction of labour with remote continuous monitoring. Midwifery 2012.

5. Adelson PL, Wedlock GR, Wilkinson CS, Howard K, Bryce RL, Turnbull DA. A cost analysis of inpatient compared with outpatient prostaglandin E2 cervical priming for induction of labour: results from the OPRA trial. Aust Health Rev 2013;37(4):467-73.

6. Ramsey PS, Meyer L, Walkes BA, Harris D, Ogburn Jr PL, Heise $\mathrm{RH}$, et al. Cardiotocographic abnormalities associated with dinoprostone and misoprostol cervical ripening. Obstet Gynecol 2005;105(1):85-90.

7. Kalkat RK, McMillan E, Cooper H, Palmer K. Comparative Study of Dinoprostone Sloe Release Pessary (Propess) versus Gel (Prostin) for Induction of Labour. J Perinat Med 2007;35(Suppl. II):S182.

8. Langley GL, Moen R, Nolan TW, Norman CL, Provost LP. The Improvement Guide: A Practical Approach to Enhancing Organizational Performance. 2nd ed. San Francisco, California, USA: Jossey-Base Publishers; 2009.

9. Hughes EG, Kelly AJ, Kavanagh J. Dinoprostone vaginal insert for cervical ripening and labor induction: a metaanalysis. Obstet Gynecol 2001;97(5):847-55.

10. Kelly AJ, Alfirevic Z, Ghosh A. Outpatient versus inpatient induction of labour for improving birth outcomes. Cochrane Libr 2013.

\section{Declaration of interests}

SO and MG have received educational grants from Ferring Limited.

\section{Acknowledgements}

The authors would like to acknowledge the many midwives, doctors, healthcare support staff, and administrative staff, who have resulted in a successful improvement project through their own dedication and team working.

In particular, other members of the project team included Ms Denise McEneaney, Ms Deborah Twyman, and Ms Rachel Parker. The authors are very grateful for their contribution and hard work.

We would also like to thank Dr Saimah Arif and Dr Andrew Rochford, for their roles as mentors and trainers in improvement methodology, and Dr Colette Smith for her assistance in the statistical analysis.

\section{Ethical approval}

According to policies set by the institution's healthcare trust, this work was exempt from ethical approval, as a non-research quality improvement aiming to provide systematic, data guided activities designed to bring about immediate improvements in health care delivery. It was registered as an audit with the trust clinical effectiveness unit. 